\title{
The last revolution of new comets: the role of stars and their detectability
}

\author{
M. Fouchard ${ }^{1,2, \star}$, H. Rickman ${ }^{3,4}$, Ch. Froeschlé ${ }^{5}$, and G. B. Valsecchi ${ }^{6}$ \\ 1 University Lille 1, LAL, 59000 Lille, France \\ e-mail: marc.fouchard@univ-lille1.fr \\ 2 Institute de Mécanique Céleste et Calcul d'Éphémérides, 77 Av. Denfert-Rochereau, 75014 Paris, France \\ 3 P.A.S. Space Research Center, Bartycka 18A, 00-716 Warszawa, Poland \\ 4 Dept. of Physics and Astronomy, Uppsala University, Box 516, 75120 Uppsala, Sweden \\ 5 UNSA CNRS UMR 6202, Observatoire de la Côte d'Azur, Bd de l'Observatoire, BP 4229, 06304 Nice Cedex 4, France \\ ${ }^{6}$ IASF-Roma, INAF, via Fosso del Cavaliere 100, 00133 Roma, Italy
}

Received 14 January 2011 / Accepted 30 August 2011

\begin{abstract}
Context. This work is a follow-up of a previous study, where we simulated the dynamical evolution of the Oort Cloud over 5 Gyr with special attention to the injection of comets into observable orbits.

Aims. We wish to clarify how comet injection operates with two types of perturbers: Galactic tides and passing stars. We illustrate why attempts to identify the stars that might have played an important role in injecting the observed new Oort Cloud comets are as yet unlikely to succeed, and investigate how large an improvement can be expected from the Gaia mission.

Methods. We simulate a 5 Gyr time span, concentrating on the injections found during the last 3 Gyr by extracting detailed information about the last revolution of the injected comets. We analyse the contributions of both the Galactic tides and the stars separately, and assess their importance as a function of the semi-major axis of the comets. We also compute the distances and motions of the perturbing stars at the time the comets reach their perihelia and thus estimate their observability.

Results. By studying more than 20000 injected comets, we determine how the likelihood of tidal and stellar injections varies with the semi-major axis. We establish the range of semi-major axis for which a real-time synergy between stellar and tidal perturbations is important. We find how many perturbing stars could be identified using HiPPARcos and Gaia data, and how the dynamics of injections would change, if only the observable stars were acting.

Conclusions. The number of injected comets peaks at a semi-major axis $(a)$ of about 33000 AU but the comets spread over a wide range around this value. The tides are unable to inject any comets at $a<23000 \mathrm{AU}$ but would be able to inject almost all of them at $a>50000 \mathrm{AU}$. The real-time synergy is found to extend between $a \sim 15000 \mathrm{AU}$ and $a \sim 45000 \mathrm{AU}$ and to be the main contributor at $a \sim 25000$ AU. Stellar perturbations make important contributions at all semi-major axes. On the basis of HiPPARcos data, only a minority of the stars that may contribute to comet injections are detectable, since most stars have escaped to distances beyond the HiPPARCos detection limit. For Gaia, on the other hand, a large majority of the perturbing stars will be both identifiable and measurable.
\end{abstract}

Key words. celestial mechanics - comets: general - Oort Cloud

\section{Introduction}

When Oort (1950) introduced the concept of a very distant source region for long-period comets (the "Oort Cloud"), he was aware of the need for an efficient mechanism to bring the perihelia of comets from the region well beyond the orbits of Jupiter and Saturn (typically, perihelion distance $q>15 \mathrm{AU}$ ) into the observable range (nowadays, $q<5 \mathrm{AU}$ ). If this does not happen during just one orbit, it is likely that the comet is lost from the process because of a planetary perturbation that either ejects it from the Solar System or captures it into a much more tightly bound orbit.

Oort (1950) identified the impulses imparted to comets by passing stars as a likely mechanism for comet injection. Under usual conditions, it would work exclusively for the orbital range of the "new comets", i.e., for semi-major axes $a>10000$ AU. Comets orbiting at closer distances would not have the time to experience a relevant perturbation during one orbital revolution. However, Hills (1981) pointed out that an Oort Cloud extend-

\footnotetext{
* Present address: Observatoire de Lille, 1 impasse de l'Observatoire, 59000 Lille, France.
}

ing inward of the above limit will from time to time be perturbed by close stellar encounters - possibly leading to large, episodic increases in the flux of new comets including smaller than usual semi-major axes. These events have been termed "comet showers".

In the mid-1980's, it was realized that the Galactic tidal force also has an important influence on comet injection, and may in fact represent the predominant effect (Duncan et al. 1987; Delsemme 1987). In particular, Heisler \& Tremaine (1986) showed that the "vertical" disk tide is an efficient perturber, causing regular $q$ oscillations in the range of $a$ of about 30 000-40 000 AU. On the basis of HiPParcos results (Perryman et al. 1997), the local density of the Galactic disk has been found to be lower than previously thought (Holmberg \& Flynn 2000) thus reducing the influence of the disk tide, but its significance remains indisputable.

In a previous paper (Rickman et al. 2008), we simulated the evolution of the Oort Cloud assuming that it is perturbed by both Galactic tides and passing stars over a time interval of up to $5 \mathrm{Gyr}$. We found that the injection of new comets at present as well as during the past few Gyr - is dominated by a synergy 
between the two perturbers. While it may be that this synergy is largely due to the stars filling the "tidally active zone", from where the disk tide may bring the comets into observable orbits (Fouchard et al. 2011, to be referred to as FFRV11), there also appears to be a more direct involvement of the stars that is quite significant at $a<30000$ AU (Rickman et al. 2008).

Another way to analyse the process of comet injection was followed by Dybczyński (2001, 2006), who took a sample of high-quality original orbits of observed long-period comets, integrating them backward to the previous perihelion passage. In his first paper, he considered only the Galactic tides and found that, while comets with original semi-major axes of $a_{\mathrm{o}}>$ $25000 \mathrm{AU}$ tend to have moved from orbits with $q>15 \mathrm{AU}$, those with smaller values of $a_{\mathrm{o}}$ have not. By performing additional integrations including the perturbing action of the Algol system during its slow encounter about $7 \mathrm{Myr}$ ago, he was able to show that a few additional comets made the jump from $q>15$ AU. He thus proposed that the inclusion of the stellar perturbations in the dynamical model would be very important in revealing which comets are indeed "new" rather than simply fulfilling a criterion based on $a_{\mathrm{o}}$.

The second paper (Dybczyński 2006) presented an improvement to the search for Hipparcos stars experiencing close encounters with the Sun in the recent past or near future, which had earlier been made by García-Sánchez et al. (1999, 2001). This may be said to represent the state of the art of the matter, and the result was a list of 23 stars identified to have passed within $2.5 \mathrm{pc}$ of the Sun during the previous 3 Myr. Only 11 of these actually penetrated to within $2 \mathrm{pc}$, which is $\sim 10 \%$ of the statistically expected total of $\sim 120$ based on an expected flux of about 10 encounters within 1 pc per Myr (García-Sánchez et al. 2001). None of the passages was found to have triggered the injection of any observed comet, and Dybczyński's conclusion was that the current comet injections are dominated by the Galactic tides.

Królikowska \& Dybczyński (2010) selected a sample of 26 comets for which high-quality original orbits could be derived while accounting for non-gravitational (NG) effects (Królikowska 2006). All these comets have original values of $1 / a_{\mathrm{o}}$ less than $10^{-4} \mathrm{AU}^{-1}$, so they are "new" in Oort's sense. For swarms of clones compatible with the observations, these authors integrated the orbits backward and forward until the subsequent perihelion passage or the ejection of the comet from the Solar System. In accordance with Dybczyński (2006), only the Galactic tides were considered as a perturbing force in these integrations. Thus, taking account of observational uncertainties as well as NG effects, they concluded that fewer than $30 \%$ of the comets are actually new in the sense of having passed from $q>15$ AU during the last revolution. This result is largely caused by non-gravitationally determined original orbits tending to have smaller semi-major axes than one obtains when neglecting the NG effects.

Dybczyński \& Królikowska (2011) performed a similar study, focusing on relatively recent comets with perihelia beyond 3 AU. They again found a large fraction of "dynamically old" comets, which apparently had their previous perihelion passage well within the "loss cylinder". Thus, the Jupiter-Saturn barrier does not appear to be as efficient an obstacle as has been thought. While the actual role of this barrier was recently investigated by Kaib \& Quinn (2009) and shown to allow the passage of comets via previously unexpected routes, it is also of interest to re-evaluate the conclusions about the insufficiency of stars to act in the current comet injections. In this paper, we investigate the roles of passing stars and the Galactic tides during the last revolution before an observable comet appears, based on our dynamical simulations.

In Sect. 2, we introduce three different hypothetical Oort Clouds containing $10^{6}$ comets perturbed simultaneously by the Galactic tides and three different sequences of stellar encounters, each sequence acting on one Oort Cloud. Section 3 is devoted to our results, paying special attention to the role of the stars in connection with that of the tides. In Sect. 4, we investigate, based on our simulations, the observability by the Hipparcos or Gaia missions of the stars that have passed during the last revolution of the currently discovered new Oort Cloud comets, and whether only those that are observable would be able to inject a sufficiently large amount of comets. Our conclusions are summarized in Sect. 5.

\section{Models and calculations}

\subsection{Models}

As in Rickman et al. (2008), we build a thermalized initial Oort Cloud of $10^{6}$ fictitious comets with orbital elements chosen at random, and we study their dynamical evolution over 5 Gyr. Each comet is subject to both tidal and stellar perturbations. The initial conditions of these fictitious comets are as follows: the semi-major axes $a_{0}$ are chosen such that $3 \times 10^{3}<a_{0}<$ $1 \times 10^{5} \mathrm{AU}$ with a probability density $\propto a_{0}^{-1.5}$ (Duncan et al. 1987), and the eccentricities $e_{0}$ are chosen with a density function $\propto e_{0}$ with the constraint that the perihelia are outside the planetary system, i.e., $q_{0}>32 \mathrm{AU}$. The initial angular elements, $\omega_{0}, \Omega_{0}$ and $M_{0}$, which may be defined with respect to an arbitrary frame of reference, are randomly chosen with a flat distribution, and the same holds for $\cos i_{0}$.

We choose three different sets of initial conditions, i.e., we consider three different initial Oort Clouds. They form three realizations of the distributions described above. We also consider three different samples of passing stars. Each sample corresponds to a sequence of 197906 stellar encounters, occurring at random times during an interval from zero to $t_{\max }=5 \times 10^{9} \mathrm{yr}$, with random solar impact parameters up to $d_{\max }=4 \times 10^{5} \mathrm{AU}$, and with random stellar masses and velocities according to the procedure described by Rickman et al. (2004, 2008). Each cloud defined previously is perturbed by one of the three stellar samples.

For the computation of the stellar perturbations acting on the heliocentric orbit of a comet, we use the "sequential impulse approximation" (Rickman et al. 2005), which employs hyperbolic deflections to compute the impulses (Dybczyński 1994) while accounting for the motion of the comet by integrating the instantaneous acceleration over time using finite steps. This method has been shown to be quite accurate even for comets moving in the inner core.

Our simulation proceeds for each comet by integrating the Galactic tidal effects as described in Breiter et al. (2007) and Fouchard et al. (2007) between successive moments of closest approach to the Sun by stars. At these moments, the tidal integration is stopped, and the orbital elements of the comet are updated using the stellar impulses.

The motions of the fictitious comets are integrated at most over 5 Gyr. However, the integration is stopped when the heliocentric distance of a comet becomes either $r>4 \times 10^{5} \mathrm{AU}$ or $r<15$ AU. The threshold of $15 \mathrm{AU}$ is a crude way to model planetary perturbations. A comet with $q<15 \mathrm{AU}$ is assumed to either be ejected into interstellar space, which means that the current perihelion passage is the last one, or be sent into a much 
more tightly bound orbit, in which case the influence of external perturbers (Galactic tides or passing stars) is effectively cut off. Each time we stop an integration on account of $r<15 \mathrm{AU}$, we take note of the actual perihelion distance and record a case of injection into an observable orbit, if $q<5 \mathrm{AU}$. We refer to this as a post-injection orbit. For every comet that is thus injected, its orbital elements at the previous perihelion passage are saved. These refer to what we shall call the pre-injection orbit.

The number of injected comets per interval of 20 Myr versus time is shown in Fig. 1. The sequences of stellar encounters are seen to be quite different, the third simulation having more frequent strong stellar encounters than the other two. This is shown by the large number of high peaks, corresponding to comet showers. However, the background fluxes are similar with a global decrease in each case. In this regard, we emphasize that all the stellar encounter sequences are constructed in the same way and are consistent with the observed number densities and velocities of stars in the current solar neighbourhood. Obviously, these data do not provide strong constraints on the history of the Oort Cloud in terms of major stellar perturbations, but we can see that the quiescent flux of comet injections is not very strongly affected by this uncertainty.

The decrease in the flux is explained by the depletion of the tidally active zone (TAZ) - i.e., the region of phase space from which the Galactic tides are able to decrease the perihelion distance below $5 \mathrm{AU}$ - with time. We describe this phenomenon in detail in FFRV11. The strong stellar perturbations that induce comet showers are efficient in re-injecting comets into the TAZ, thereby influencing the flux of injected comets during several hundred Myr hence, but they also accelerate the loss of comets from the "central part" of the cloud (intermediate range of semimajor axes), where most of the injections occur. That the background flux behaves similarly in the three simulations in spite of the different numbers of shower-inducing events can thus be understood by considering that the flux of injections is proportional to both the number of comets in the central Oort Cloud and the relative amount of TAZ filling in this zone. With a larger number of shower-inducing stellar encounters, the first factor decreases while the second one increases.

At the beginning of the integrations, the TAZ is full. Because this situation is rather exceptional the first 2 Gyr of each integration will not be taken into account (see FFRV11). This 2 Gyr time span is more or less the time needed for the central Oort Cloud to settle into a normal state of TAZ filling.

\subsection{The comet enhancements}

The high peaks seen in Fig. 1 are indicative of comet showers caused by specific stellar encounters. The comets injected during a shower have different characteristics in terms of their number and distribution of orbital elements than the comets injected during a quiescent period. For instance, the high rate of injections that characterizes comet showers is often due to the rarely seen passage of a star through the inner core of the Oort Cloud. This causes lots of injections from this inner core (small values of the semi-major axis), which is more or less immune to injections under normal circumstances. Consequently, we need to take care to separate the injected comets into those that experienced quiescent conditions and those that might have suffered an abnormally large stellar perturbation. In particular, we aim to use the set of quiescent comets as a proxy for the current conditions in order to draw conclusions about the injection of the observed "new comets".
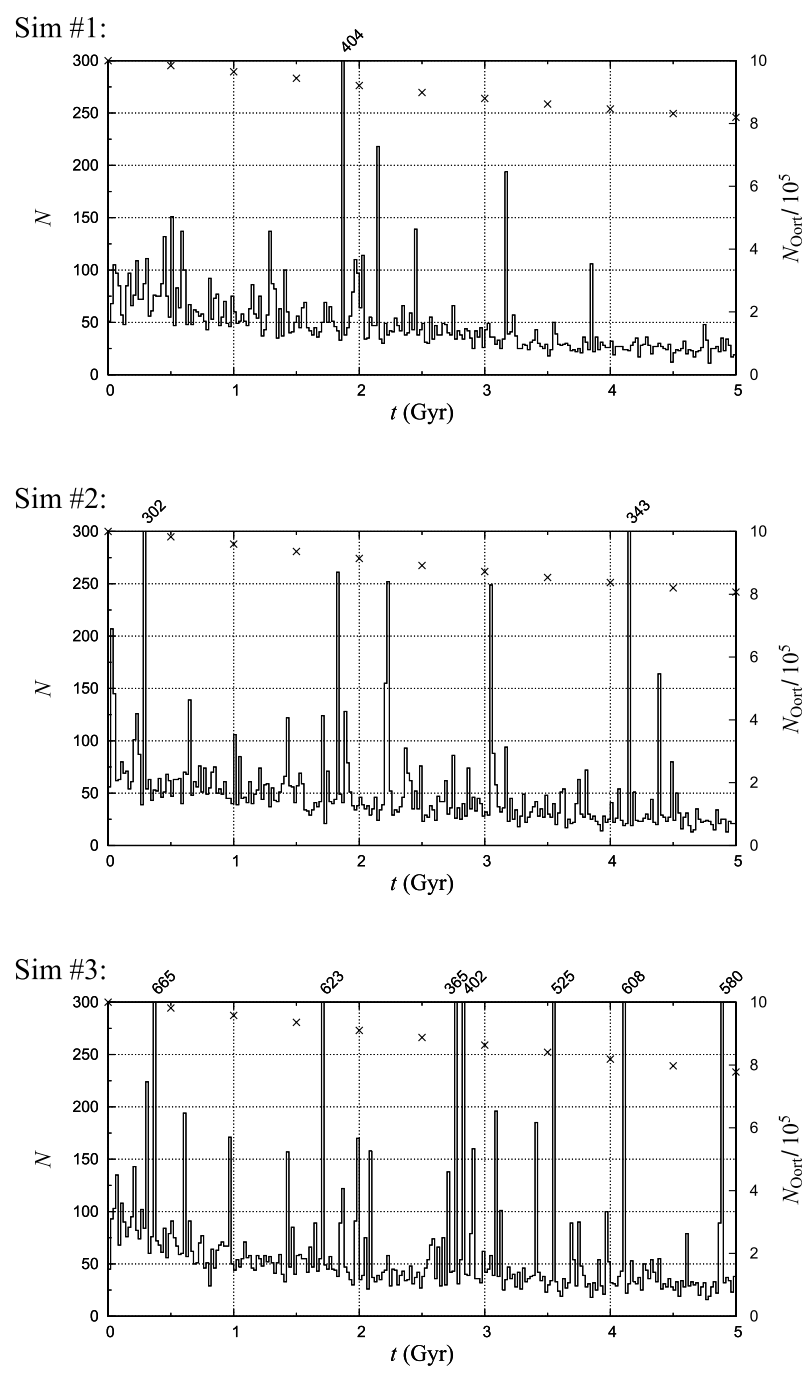

Fig. 1. Number of observable comets per interval of 20 Myr versus time for the three simulations. When the number exceeds 300 , it is written above the respective graph. The crosses give the number of comets in the Oort Cloud as counted every $500 \mathrm{Myr}$ (scale to the right).

The problem is how can we define a comet shower? Such an event is due by definition to a single star. Hence, the best approach is to assess, for each star, whether the star is capable of inducing a large enhancement of the rate of comet injections or not. To this aim, we use the results of the simulations described in FFRV11, which tell us that, using $\simeq 10^{6}$ sample comets, the number $N_{\star}$ of injected comets, following a single stellar encounter, is statistically approximated by ${ }^{1}$

$N_{\star}=\left(\frac{M_{\star}}{V_{\star} \sqrt{d_{\odot}}}\right)^{1.82} \times 16.23$

for low-mass stars, and

$N_{\star}=\left(\frac{M_{\star}}{V_{\star} d_{\odot}^{2}}\right)^{0.89} \times 12.83$,

for high-mass stars. The units for $M_{\star}$ (stellar mass), $V_{\star}$ (stellar speed at infinity), and $d_{\odot}$ (impact parameter with respect

1 These two power laws were obtained from a different initial Oort Cloud, i.e., with a flat distribution of orbital energy. It is, however, quite close to our simulated initial Oort Cloud, also because the distribution of orbital energy evolves with time, as we see later. Consequently, we apply these power laws to our simulated Oort Cloud. 
to the Sun) are, respectively, the solar mass, $40 \mathrm{~km} \mathrm{~s}^{-1}$, and 20000 AU. High-mass stars were found to be those with $M_{\star}>2$ and low-mass stars those with $M_{\star} \leq 0.9$. The two formulae are relevant for close encounter perturbations and distant, tidal perturbations, respectively (FFRV11). Stars of intermediate masses would likely act in either way, depending on the detailed circumstances.

In this investigation, we use the term comet enhancement for cases when a star passes with $N_{\star}>5$. For each star of our simulations, we know $M_{\star} / V_{\star}$, and using Eqs. (1) and (2) one can derive two values of $d_{\odot}^{(c)}$ (one per equation) such that $N_{\star}>5$ for $d_{\odot}<d_{\odot}^{(c)}$. If $M_{\star} / V_{\star}=0.60077$, both values of $d_{\odot}^{(c)}$ are equal, amounting to 1.316 (i.e., $26320 \mathrm{AU}$ ). In general, when selecting the value of $d_{\odot}^{(c)}$ to use, we always take the smallest one, i.e., the most restrictive definition of enhancement-producing stars.

In terms of the 13 stellar types that we consider, the most common ones (red and white dwarfs) predominantly have $M_{\star} / V_{\star}<0.60077$, which means that we identify the enhancement makers among such stars using Eq. (1) - as indeed we should (FFRV11). For all the other types (giants and mainsequence stars down to K0), we mostly use Eq. (2). Judging from Fig. 1 of FFRV11, this choice is actually relevant - not only for the high-mass stars defined above but also for the lower mass types.

We subdivide the injected comets into two categories according to whether or not there was any passage by an enhancementproducing star during the last revolution of the comet. We use the term quiescent comets strictly for those comets that did not experience any such stellar passage, and all the others will be described as non-quiescent comets. Only the first category will be used as a proxy for the observed new comets.

We note that in our simulations, two comets injected into simultaneous perihelion passages may actually belong to different categories, because their last revolutions may have taken different amounts of time. Thus, the comet with the shorter period may fulfil the requirement of being quiescent, while the other one does not. Hence, we do not consider comet enhancements in terms of specific time intervals. We use instead "quiescent" and "non-quiescent" as flags of injected comets indicating whether their last orbit may or may not have been influenced by a star with a high injection efficiency ${ }^{2}$.

With the above criteria, we have a total of 1227 enhancement-producing stars out of a total of 593718 passing stars during the full length of all three simulations. During the last $3 \mathrm{Gyr}$, the number of enhancement-producing stars is 755 out of a total of 355821 passing stars. While this fraction is very small, the number of enhancement makers is much larger than the number of high peaks in Fig. 1. Thus, we can expect all high peaks (major showers) to be caused by enhancement makers, and conversely, the stars that do not cause enhancements also not to cause any significant peaks.

From Fig. 1 of FFRV11, one can see that the number of injections caused by non-enhancement-producing stars is never much larger than 10 for an Oort Cloud of the same size as the current ones. This confirms the above expectation. Moreover, some enhancement-producing stars actually produce no comet injections at all, even though the statistical expectation is at least 5. We conclude that our definition of enhancement-producing stars is rather liberal, and many comets that we consider as nonquiescent actually behave in the same way as the quiescent ones.

\footnotetext{
2 Note that a non-quiescent comet does not have to be strongly perturbed by the enhancement-producing star. Its injection may well have been caused by the Galactic tides or by another star.
}

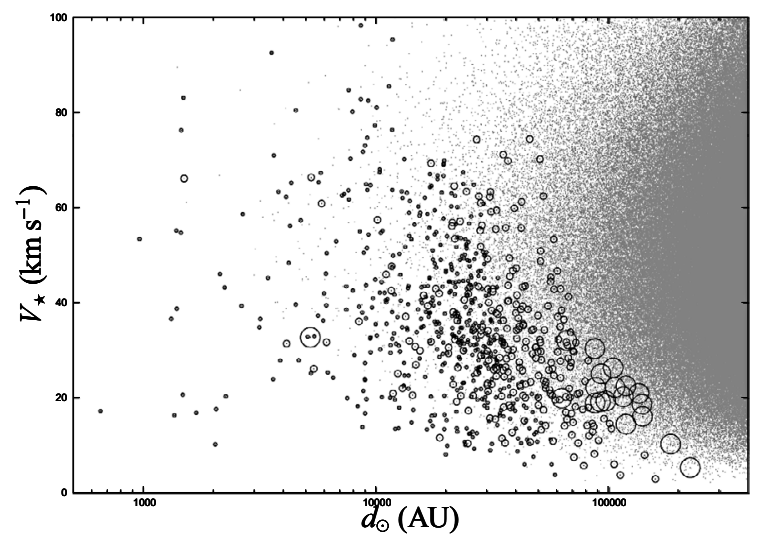

Fig. 2. Velocity at infinity versus solar impact parameter for all the stars passing during the last $3 \mathrm{Gyr}$ of the simulations. Tiny, grey dots correspond to stars that do not cause enhancements, black dots to enhancement-procucing stars with $M_{\star}<1.2$, small black circles to enhancement-producing stars with $1.2<M_{\star} \leq 4$, and large black circles to enhancement-producing stars with $M_{\star}=9$ (B0 stars).

If the number of injections by a certain star in our simulations were 5-10, the corresponding number for the real Oort Cloud in case this contains $10^{12}$ comets would be $\sim(5-10) \times$ $10^{6}$. Over an interval of several million years, the flux of new comets with $q<5$ AU would only be a few per year, which would be barely noticeable as an increment upon the estimated flux of 0.8 comets per year per AU of perihelion distance after correction for discovery bias (Francis 2005). Thus, what we call enhancements are not comparable to the comet showers discussed in previous literature. They are much less significant, so our criterion for defining quiescent comets is indeed conservative.

Such conservatism is of relevance, since the Solar System is statistically unlikely to be experiencing a major comet shower at the moment (Dones et al. 2004), and the orbital distribution of long-period comets does not show the expected features of a shower (Dybczyński 2002). Hence, the observed new comets are most likely quiescent ones, and in order for our model to be applicable to these, our quiescent comets should indeed be quiescent, i.e., unaffected by any significant enhancement.

Figure 2 plots the velocity at infinity $V_{\star}$ versus the solar impact parameter $d_{\odot}$ for stars passing during the last $3 \mathrm{Gyr}$ of all three simulations. The masses of the enhancementproducing stars are indicated by different black symbols, while all other stars are shown by tiny, grey dots. As expected, the enhancement-producing stars are stars with small impact parameter and/or low velocity as a general feature. However, the higher the mass of the star, the larger the impact parameter is allowed to be. In particular, the highest-mass stars may produce enhancements at almost any impact parameter, if their velocity is low enough.

We have a total of 20446 injected comets during the last $3 \mathrm{Gyr}$, among which $29.9 \%$ are of the quiescent type and $70.1 \%$ are non-quiescent. During the first $2 \mathrm{Gyr}$, we had 21385 injected comets, out of which $36.7 \%$ were quiescent. The higher proportion of quiescent comets initially is due to the TAZ being more populated, which facilitates the role of the tides in injecting comets. As mentioned above, this is the reason why we study only the comets injected during the last $3 \mathrm{Gyr}$. 


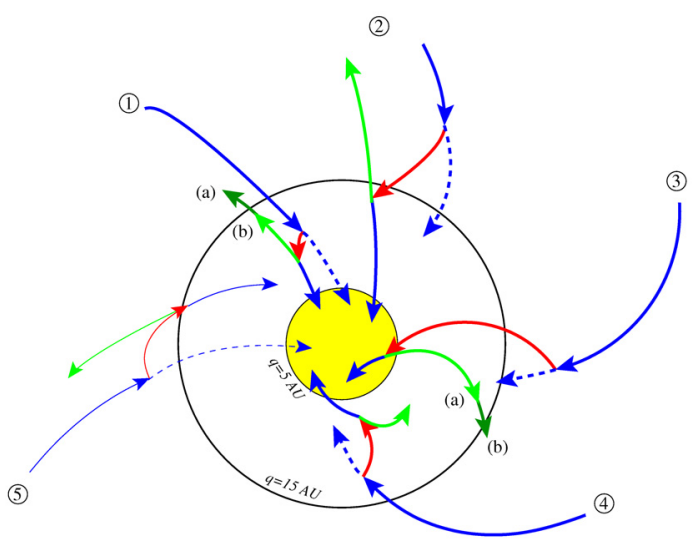

Fig. 3. Schematic representation of the variation in the angular momentum for different injection scenarii. The distance to the centre in this diagram represents the angular momentum of the comets, i.e., the perihelion distance in the present case of quasi-parabolic orbits. The full black circle corresponds to a perihelion distance of $15 \mathrm{AU}$, and the yellow disk corresponds to perihelion distances smaller than $5 \mathrm{AU}$. The meaning of the arrows is explained in the text. For each trajectory, the comet starts at perihelion before its last revolution.

\subsection{Injection scenarii}

Before explaining our results we need to describe the different main scenarii for the last orbital period of injected comets. To begin with, we note that stellar perturbations are rarely negligible - thus the pre- and post-injection orbits are rarely connected by a single tidal trajectory. As illustrated in Fig. 3, there are not very many cases to distinguish. The figure shows the main features of dynamical evolutions leading to comet injection during the last orbit before the observable perihelion passage. We note that it does not cover all possible scenarii but has been simplified for clarity.

We represent the generally decreasing trend of perihelion distance associated with injections by arrows directed toward the centre. The yellow region denotes the observable orbits, and the white, surrounding one represents the Jupiter-Saturn barrier. The red and blue arrows show the evolution - forwards in time - caused by stellar impulses and the Galactic tides, respectively. By dashed blue arrows, we indicate how the tidal perturbation would have continued to act in the absence of the stellar impulse. The green arrows show the backward evolution starting from the time of the stellar perturbation, if only the tides are allowed to act. We assume for simplicity that there is only one significant stellar impulse during the last revolution of the comet.

The cases numbered 1-4 represent the vast majority of all injections. Case 1 refers to tidal injections, where stars play a relatively insignificant role. They may perturb the comets, thus affecting somewhat the post-injection orbits, but even in their absence the initial trajectory would lead to an injection because of the tides. We distinguish two subclasses called $a$ and $b$, depending on the outcome of a backward integration with only tides. In case $1 a$, the comets cross the barrier into orbits with $q>15 \mathrm{AU}$, while in case $1 b$ they do not. Case 2 is different, since the injection would have failed in the absence of the stellar impulse. However, the star does not inject the comet by itself - it is only a helper to the tides.

Case 3 is again different, because now the star actually performs the injection, and the tidal action is generally rather insignificant. We may again distinguish two subclasses in the same way as in case 1 . Thus, case $3 b$ refers to comets that get injected by a stellar impulse but would appear to have been tidally injected as judged from a purely tidal backward integration. As previously mentioned, this is a relatively rare phenomenon. Case $3 a$ is the more common one, where the injected comets bear no traces of tidal injection. Finally, in case 4 an injection is achieved, but it is impossible to ascribe it to either stars or tides.

Case 4 may also be considered as a real-time synergy between the stars and tides, since these two mechanisms interact in a constructive way to ensure that the comets are injected. In reality, however, it must also happen that an injection, which the tides alone would have achieved, fails because of a stellar impulse. This is indicated as case 5 by thinner arrows, since our normal simulation does not register such outcomes. Nonetheless, we did investigate these outcomes, as described in the next section.

We now define three sets of injected comets:

- set $\mathbb{G}$ consists of those for which a backwards integration using only the Galactic tides yields a previous perihelion distance larger than $15 \mathrm{AU}$. In other words, these are comets whose post-injection orbits would have been reached by tidal injections in the absence of stars. This set involves cases $1 a$, 2, and $3 b$ of Fig. 3;

- set $\mathbb{G}^{\prime}$ contains those for which the forward integration yields a perihelion distance smaller than $5 \mathrm{AU}$ even when turning off the stellar perturbations during the last revolution. In other words, the pre-injection orbits would lead to tidal injections in the absence of stars. This set involves case 1 of Fig. 3;

- set $\mathbb{S}$ contains the injected comets for which there is one passing star during the last revolution that induces a crossing of the Jupiter-Saturn barrier. This set is represented by case 3 of Fig. 3.

These three sets intersect to some extent. For instance, comets that follow trajectory $1 a$ belong to both $\mathbb{G}$ and $\mathbb{G}^{\prime}$, whereas case $1 b$ comets belong only to $\mathbb{G}^{\prime}$ and case 2 comets belong only to $\mathbb{G}$. Moreover, there are injections that do not belong to any of the sets, as illustrated by case 4 of Fig. 3 .

\section{General results}

\subsection{The injected comets}

Among the total of 20446 comets injected during the last $3 \mathrm{Gyr}$ of our simulations, 9642 comets belong to the $\mathbb{G}$ set, out of which $5107(52.9 \%)$ are non-quiescent, and 8915 comets belong to the $\mathbb{S}$ set, out of which $8257(92.6 \%)$ are non-quiescent. We note that 739 comets belong to both $\mathbb{G}$ and $\mathbb{S}$ sets, and out of these $624(84.4 \%)$ are non-quiescent. Finally, 2628 injected comets belong to neither $\mathbb{G}$ nor $\mathbb{S}$. Out of these, about $60 \%$ are non-quiescent.

It is well-known that both tidal and stellar perturbations depend strongly on the semi-major axis of the comets, and in Fig. 4 we show the effect of these dependences on the injection statistics in terms of our $\mathbb{G}$ and $\mathbb{S}$ sets. For this purpose, we divided the range of the semi-major axis (i.e., [3000, 100 000] AU) into 150 bins such that the comets are initially equi-partitioned among the bins. For each interval, we show the number of injected comets $\left(n_{\text {inj }}\right)$, as well as the fractions $p_{\mathbb{G}}$ and $p_{\mathbb{S}}$ of comets belonging to sets $\mathbb{G}$ and $\mathbb{S}$, respectively, among the injected comets. This is done separately for quiescent and non-quiescent comets.

From top to bottom, the first four panels of Fig. 4 show the behaviour of $n_{\text {inj }}, p_{\mathbb{G}}, p_{\mathbb{S}}$ and $p_{\mathbb{S}}+p_{\mathbb{G}}$ versus the semi-major axis 
A\&A 535, A86 (2011)

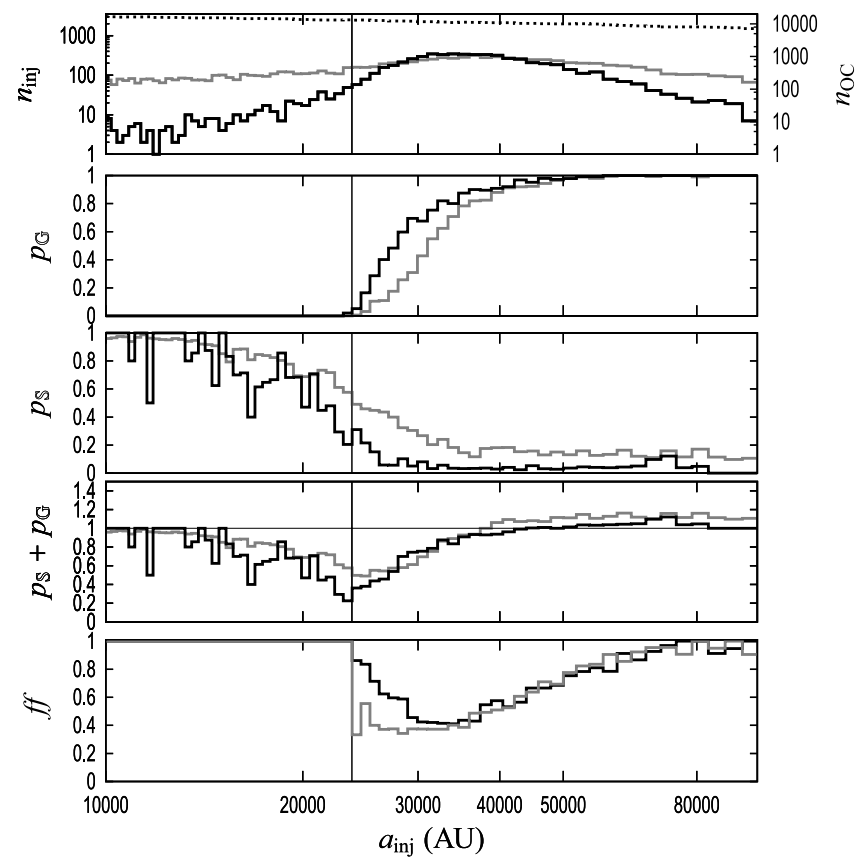

Fig. 4. The first four panels show, from top to bottom: the number of injected comets, the fraction of injected comets in set $\mathbb{G}$, the fraction in set $\mathbb{S}$, and the sum of these two fractions, all plotted versus the semi-major axis at injection $\left(a_{\text {inj }}\right)$. In these plots, the full black lines correspond to quiescent comets and the full grey lines to the non-quiescent comets. The dotted black line in the uppermost plot shows the number of comets (given on the right axis) in the Oort Cloud at $3 \mathrm{Gyr}$. The horizontal grey line in the bottom plot indicates the value of unity for the sum of the two fractions. The fifth panel shows the fraction of injected comets that are not injected any more, if one removes the stellar perturbations during the last revolution (black line), and the fraction of comets injected in a tides-only model that are not injected any more, when one adds the stellar perturbations (grey line). In all the plots, the vertical dashed line indicates the threshold $a_{\text {crit }}$, below which no comets belong to the $\mathbb{G}$ set.

at injection $\left(a_{\mathrm{inj}}\right)$. The fifth panel is different, as we describe it later.

Because we consider only the last three gigayears, the distribution of comets in the Oort Cloud has changed from the initial state. Some comets have left the cloud, and some have migrated between different ranges of semi-major axis. To illustrate the effect of the population of the Oort Cloud at injection, the distribution of the semi-major axis at $3 \mathrm{Gyr}$ is plotted in the upper panel. Initially, the number of comets in each bin was close to 20000 , which is situated at the top of the plot. We see that some depletion has occurred and that this depletion gets stronger for more distant parts of the cloud. The outermost parts have been depleted by more than a factor of two, while the innermost parts are left practically unchanged. At times later than $3 \mathrm{Gyr}$, which we consider, these depletions will have grown further.

Our results on the number of injected, quiescent comets are in general agreement with the location of synthetic Oort spikes found by others using similar experiments (e.g., Wiegert \& Tremaine 1999; Emel'yanenko et al. 2007). Our maximum of the injection efficiency occurs around $33000 \mathrm{AU}$. The decrease in $n_{\text {inj }}$ toward smaller semi-major axes is due to the wellknown decrease in the average size of both tidal and stellar perturbations. For larger semi-major axes, the decrease is partially explained by the strong depletion of the outer parts of the Oort Cloud that we commented on above. However, the main reason is that the Galactic tides change the perihelia of outer Oort Cloud comets very rapidly. The timescale is shorter than the orbital period. Thus, under the action of the tides alone, the probability for a comet to reach its perihelion when the perihelion distance is below $5 \mathrm{AU}$, i.e., the ratio of the time spent with $q<5$ AU to the orbital period $P$, behaves as $P^{-2}$ (Fouchard et al. 2010). For stellar injections, the same problem arises, i.e., the tides are likely to remove the comet from the observable zone, if there is sufficent time. Consequently, even though the number of potential comets to be injected increases with $a$ because of the growing size of the perturbations, this effect is more than offset by the aforementioned $a^{-3}$ dependence.

We note that for non-quiescent comets, the injection efficiency is almost flat with respect to the semi-major axis. This is consistent with previous results (Rickman et al. 2008) because during a comet shower the loss cone is filled at almost any semimajor axis. We conclude that, even though this category has been very liberally defined, it terms of numbers it is dominated by shower comets.

The smallest semi-major axis of the $\mathbb{G}$ set at injection is $23774 \mathrm{AU}$. We interpret this to mean that below $a_{\text {crit }} \simeq$ $23000 \mathrm{AU}$ the tides are unable to inject comets on their own. However, for larger $a_{\text {inj }}$ the $\mathbb{G}$ set fraction of injected comets rises rapidly, especially for quiescent comets, so that for $a_{\mathrm{inj}}>$ $50000 \mathrm{AU}$ it is almost unity for both categories. This illustrates the rapidly growing efficiency of tidal perturbations, which eventually even acts to quench the injection rate, as discussed above.

In the range where the rate of quiescent comet injections is at its maximum, we have $p_{\mathbb{G}}=0.812$, implying that for almost $20 \%$ of the injected comets stars must have played important roles. In some cases, these may have been as in case $1 b$ of Fig. 3, and in other cases as in cases $3 a$ or 4 . This fraction increases for smaller semi-major axes, reaching $33 \%$ for $a_{\text {inj }}=30000 \mathrm{AU}$ and almost $85 \%$ for $a_{\text {inj }}=25000 \mathrm{AU}$. We thus observe that comet injections in the inner part of the Oort peak are generally governed by stellar perturbations.

The fraction of $\mathbb{S}$ set comets decreases continuously from unity for $a_{\mathrm{inj}} \sim 10000 \mathrm{AU}$ to very small values for $a_{\mathrm{inj}}>$ $40000 \mathrm{AU}$. For quiescent comets in the latter range, $p_{\mathbb{S}}$ is always below 5\%, and even for non-quiescent comets it remains below $20 \%$, owing to the very efficient tidal perturbations in the outer part of the Oort Cloud. As we see in the next subsection, large stellar perturbations of $q$ are common for these comets, but most stellar injections are sabotaged by subsequent tidal increases in $q$, as mentioned above. On the other hand, for comets that are indeed injected - regardless of the mechanism - a backwards integration with the Galactic tides is almost sure to bring the perihelion beyond $15 \mathrm{AU}$, so that the comets will be counted with the $\mathbb{G}$ set. Thus, the tides appear to dominate the injections at large semi-major axes because of our definitions of the $\mathbb{S}$ and $\mathbb{G}$ sets. Stellar perturbations may play important roles in the game, but they are unlikely to get credit.

For quiescent comets at the other end of the $a$-range, we note that for $a_{\text {inj }} \simeq a_{\text {crit }}$, we have $p_{\mathbb{S}}=30 \%$, and for $a_{\text {inj }}=15000 \mathrm{AU}$, $p_{\mathbb{S}} \simeq 85 \%$. Hence, for this range of semi-major axes, the sum of the $\mathbb{S}$ and $\mathbb{G}$ fractions is less than unity, as shown in the fourth panel of Fig. 4, so the injections are often due to neither the tides nor any single star alone. We are dealing instead with a synergy between the tides and stellar perturbations, which acts "in real time", i.e., the tides and stars collaborate in making comets cross the Jupiter-Saturn barrier. The plot shows that this synergy reaches a maximum for $a_{\text {inj }} \simeq a_{\text {crit }}$, where the sum of the two fractions barely reaches $30 \%$, and the synergy is at work over the whole range $a_{\text {inj }} \in[15000,45000]$ AU. What happens is illustrated by case 4 of Fig. 3 . 
Finally, for $a_{\text {inj }}>45000 \mathrm{AU}$, we observe the opposite behaviour, namely, a "duplication of effort" of tides and stars with $p_{\mathbb{S}}+p_{\mathbb{G}}>1$. A star injects a comet into an orbit that could also have been reached by a tidal injection. This relatively rare phenomenon is illustrated by case $3 b$ of Fig. 3. As already mentioned, it is almost impossible for an injected comet of the outer Oort Cloud not to belong to the $\mathbb{G}$ set, so the stellar injections that do not get sabotaged will generally be assigned double membership.

To summarize the peculiarities of comet injection from the outer part of the Oort Cloud, we have seen that the very rapid tidal changes in the perihelion distance (timescale shorter than the orbital period) is an obstacle to injection owing to the difficulty in timing the perihelion passage. Another problem is that the concept of a tidally active zone (FFRV11) is no longer valid in the sense of a phase space region permanently linked to the observable orbits by tidal trajectories. We are dealing with a loosely defined set that in the presence of stars is neither filled nor empty, but whose occupation is continuously changing, involving different comets at different times. Moreover, in the case of stellar injections, we later demonstrate that the largest ones occurring per orbital period are much larger than the "target", i.e., the interval $0<q<5 \mathrm{AU}$. Thus, the target is difficult to reach, and overshoots will be the rule.

We now attempt to determine the difference between the injection rates from the Oort Cloud, if we include or exclude the stellar perturbations during the last revolution of the comets. We have seen that these perturbations may both cause and prohibit injections, and we now wish to compare these two effects. For the triggering effect, we can use the sample of injected, quiescent comets during the last $3 \mathrm{Gyr}$ of our simulations and calculate the fraction that are no longer injected, if we remove the stellar perturbations during the last revolution. For the prohibiting effect, we consider the injected comets during the first 2 Gyr in a different simulation using only the Galactic tides, and we calculate the fraction that are no longer injected after stellar perturbations are added during the last revolution.

The bottom panel of Fig. 4 plots these two fractions versus the semi-major axis at injection. Since the tides are unable to inject comets for $a<a_{\text {crit }}$, the stellar triggering effect is obviously at work in this range, while the prohibiting effect is not defined. Between $a_{\text {crit }}$ and $36000 \mathrm{AU}$, the triggering effect is clearly larger than the prohibiting one. The largest difference is obtained for $a_{\text {inj }} \sim 25700 \mathrm{AU}$, where the star-triggered fraction amounts to nearly $71 \%$, while the prohibited one is only $40 \%$.

Above $a \simeq 36000 \mathrm{AU}$, the two effects balance and grow to unity in the outer Oort Cloud. In this region, we have seen that the injections appear to be mainly tidal. However, as noted above comet injection is actually a delicate balance, and stellar perturbations are so important that injections with and without stars are completely different matters. If injection happens when stars are included, it likely vanishes when the stars are excluded, and conversely, if it happens without the stars, it likely disappears when the stars are added.

The upper panel of Fig. 5 shows the appearance of our simulated Oort peak for quiescent comets as a histogram of the $1 / a$ distribution. Different colours are used to denote the contributions of the $\mathbb{G}$ and $\mathbb{S}$ sets as explained in the figure caption. In white, we see the extent of the synergistic contribution with a maximum around $30000 \mathrm{AU}$. The $\mathbb{S}$ set injections are predominant at small semi-major axes, but overall they contribute only a small fraction and disappear completely beyond $60000 \mathrm{AU}$. The G set dominates the peak at $a>30000 \mathrm{AU}$, but we have to point out two features. The first is that this is due to the phenomenon
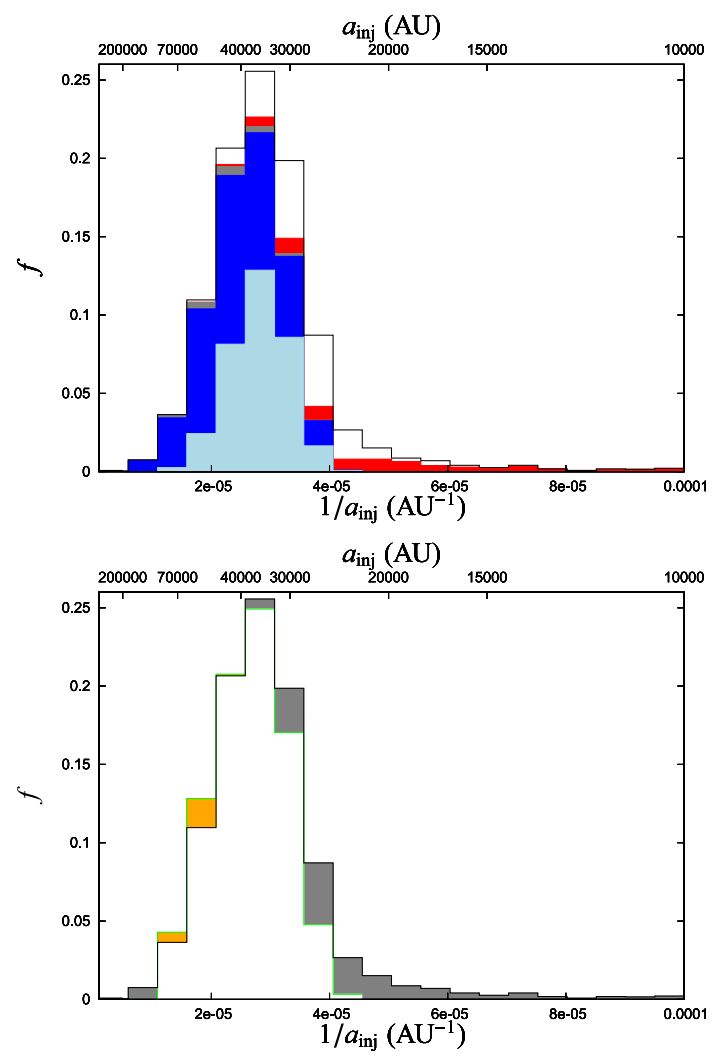

Fig. 5. Fraction $f$ of quiescent injected comets with respect to the total number (6119) plotted as a histogram versus the inverse of the semi-major axis at injection (the upper horizontal scale gives the corresponding semi-major axis). Two percent of the injected comets have $a_{\text {inj }}<10000 \mathrm{AU}$ and fall to the right of the diagram. In the upper panel, the dark-blue area corresponds to comets belonging exclusively to set $\mathbb{G}$, the sky-blue area corresponds to comets belonging to sets $\mathbb{G}$ and $\mathbb{G}^{\prime}$ but not to set $\mathbb{S}$, the grey area corresponds to comets belonging to sets $\mathbb{G}$ and $\mathbb{S}$, the red area corresponds to comets belonging to only set $\mathbb{S}$, and the white area to comets belonging to neither set. In the lower panel, the green line gives the estimated fraction of injections when the stellar perturbations are turned off during the last revolution. The orange and grey areas correspond to the excess and shortage, respectively, of the number of injections in this case.

of TAZ filling, for which massive stars are largely responsible (see FFRV11). The second is what we drew attention to above, namely, that the injections in the outer Oort Cloud are caused by an intricate interplay between the tides and the stars such that we cannot ascribe the main cause to either one alone.

In the lower panel, we attempt to compare the triggering and prohibiting effects of stars during the last revolution. The black histogram is the same as in the upper panel. We can correct the fraction of comets in the $\mathbb{G}^{\prime}$ set by increasing it using the percentages of tidal injections prohibited by stars as plotted in the bottom panel of Fig. 4. This enables us to get an estimate of the number of injections, shown by the green histogram, for the case in which the stellar perturbations were turned off for the last revolution. The orange area corresponds to the stellar prohibiting effect and the grey one to the stellar triggering effect. We see that in the outer part of the Oort peak there is a range where the stellar prohibiting effect slightly dominates but this is more than compensated for by the predominance of the triggering effect in the inner part of the peak and the region inside it.

We emphasize that only the sky-blue area in the upper panel of Fig. 5 corresponds to comets that are essentially injected by 

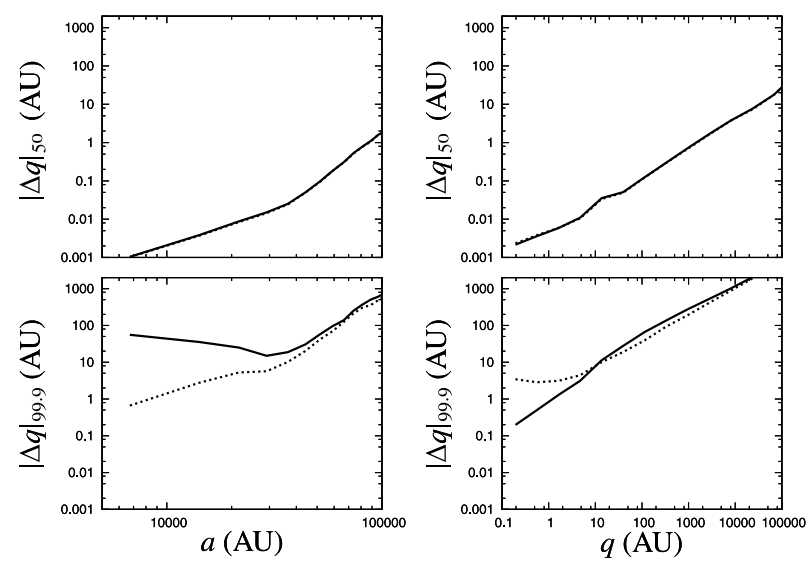

Fig. 6. Medians and 99.9th percentiles of the absolute value of the perturbation of perihelion distance. Solid curves correspond to negative perturbations and dotted curves to positive perturbations. The upper plots show the median versus the semi-major axis (left) and versus the perihelion distance (right). The lower plots show the same for the 99.9th percentile.

the tides so that stellar perturbations are of no consequence. Since this area amounts to a total of only $\sim 1 / 3$ of the injections and never exceeds $1 / 2$ of the injections in any bin of the histogram, we conclude that the action of stars during the last revolution is critically important for most new comets injected from the Oort Cloud.

We note that the distribution shown in Fig. 5 is a long-term time average, and it is worth asking, whether one can expect a nearly continuous flux of comets across the whole range of $1 / a$ that we have plotted. Taking $a=10000 \mathrm{AU}$ as an example, and requiring a rather close stellar encounter to accomplish the injection of such a comet, we find that a solar encounter distance of $d_{\odot} \sim 20000 \mathrm{AU}$ is needed. Such encounters are expected at the rate of one per $10 \mathrm{Myr}$ approximately, so even though it may be that a star injects comets that arrive at perihelion over an extended period of time, we cannot expect a truly continuous flux. Moreover, according to Fig. 2, most stars that pass at such a close distance are enhancement-producing stars, which we do not consider in Fig. 5. We conclude that the whole far tail extending to the right of the Oort peak is of sporadic occurrence and should not necessarily be represented in the sample of observed new comets.

\subsection{The role of stars}

In Fig. 6, we show the behaviour of the median and the 99.9th percentile ( 1 perturbation out of 1000 is larger than this) of $|\Delta q|$ for positive and negative stellar perturbations versus $a$ and $q$. The sample in question is the 20446 injected comets that we discussed above - both quiescent and non-quiescent ones. The number of individual perturbations during the last revolutions is about $5 \times 10^{6}$.

The medians of the negative and positive perturbations behave identically versus $a$. The curve shows a break in the slope for $a \sim 40000 \mathrm{AU}$, which we relate to, based on previous results, stellar perturbations for smaller semi-major axes being important to comet injection, while for larger semi-major axes the Galactic tides would be perfectly capable of injecting comets on their own.

Similarly, we see a strong asymmetry of the 99.9th percentiles building up and increasing with decreasing $a$ for $a<40000 \mathrm{AU}$. The values are much larger for negative

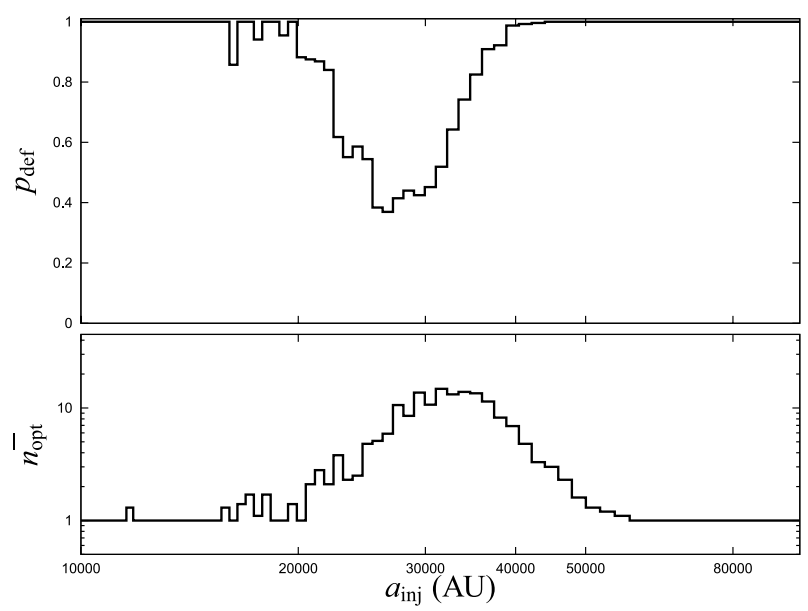

Fig. 7. Upper plot: fraction ( $\left.p_{\mathrm{def}}\right)$ of quiescent comets for which $n_{\mathrm{opt}}$ is defined versus $a_{\text {inj. }}$. Lower plot: mean $\overline{n_{\mathrm{opt}}}$ versus $a_{\mathrm{inj}}$ for the same set of comets.

perturbations, because these are the ones that inject the comets. The positive perturbations obviously have nothing to do with the injections and thus are not biased toward large values by this selection. The increase in the negative values toward smaller $a$ flattens out at $a \sim 15000 \mathrm{AU}$, where the injections start to be completely dominated by stellar perturbations.

That this asymmetry is more or less absent for $a>$ $40000 \mathrm{AU}$ tells us that the injected comets in this range are typical of comets in general and do not require exceptionally large stellar perturbations. The number of stellar encounters during the last revolution is indeed generally larger than 300 , so the 99.9th percentile should be a rough estimate of the largest individual perturbation experienced by an injected comet. We thus see that those comets tend to have experienced very significant stellar perturbations of $q$, amounting to tens or hundreds of AU.

The larger values of the 99.9th percentile for positive perturbations found at the smallest perihelion distances demonstrate the effect already noted by Öpik (1932), namely that stellar perturbations preferentially lead to an increase in the perihelion distance, when the initial orbit - in a similar way to those of meteors and observed comets - has a small perihelion distance. The reason is obvious, i.e., a large negative change in the angular momentum along the vector itself, when the absolute value is small to begin with, will increase this absolute value (and thus the perihelion distance) while reversing the sense of motion. We indeed see that the largest negative perturbations (as approximated by the 99.9th percentile) are exactly equal to the initial perihelion distance, while the largest positive ones are much larger.

We now consider whether the stellar influence on comet injections is generally due to only one star or to several stars. We investigate this by considering for each quiescent injected comet all the stars that perturbed its motion during the last revolution and counting the minimum number of stars needed to build up a total decrease in $q$ larger than 10 AU (corresponding to a jump across the Jupiter-Saturn barrier). We call this number $n_{\mathrm{opt}}$, where "opt" stands for optimistic, because there is no guarantee that the perturbations in question actually led to a crossing of the barrier.

Figure 7 plots, versus $a_{\text {inj }}$, the fraction $p_{\text {def }}$ of quiescent comets for which $n_{\text {opt }}$ is defined (i.e., for which it is possible to reach a decrease in $q$ by more than 10 AU by adding individual perturbations) and, for those comets, the mean $\overline{n_{\mathrm{opt}}}$. 
The fraction for which $n_{\text {opt }}$ is defined is less than unity only for $a_{\text {inj }}$ between 16000 and $44000 \mathrm{AU}$ - the same interval where the direct synergy in comet injection was noted. This fraction indeed follows almost exactly the behaviour of $p_{\mathbb{S}}+p_{\mathbb{G}}$ as shown in Fig. 4. From 16000 AU to $a_{\text {crit }}$, there are many comets for which neither the tides nor the stars are able to perform the injections on their own, so that the synergy is strictly necessary. In addition, while it appears that a single star may be responsible for injections below $a \sim 20000 \mathrm{AU}$, for larger semi-major axes several stars usually contribute.

Below $a=16000 \mathrm{AU}$ and above $58000 \mathrm{AU}$, we have strictly $\overline{n_{\mathrm{opt}}}=1$, so that the injections might be due to a single star. While this is certainly the case below 16000 AU, since Fig. 4 shows that $p_{\mathbb{S}} \simeq 1$, we know that above 58000 AU the situation is quite different. Figure 6 provides an indication that stellar perturbations are systematically strong for such large semi-major axes, and if there had been no Galactic tides, they would certainly have injected comets on their own. However, we saw in connection with Fig. 4 that the same can be said of the Galactic tides. As discussed above, each comet injection is indeed likely caused by the interplay of both perturbers and would disappear if either of them were removed.

\section{Star detection by HIPPARCos and Gaia}

In the previous section, we saw that stellar perturbations play an important and direct role in comet injections from all parts of the Oort Cloud, and that this role is crucial for semi-major axes smaller than about 25000 AU. Figure 6 (lower left panel) shows that stellar perturbations leading to a significant decrease in $q$ occur in general for injected comets. However, in the Introduction, we referred to the work of Dybczyński (2006), which concluded that none of the observed stars that passed near the Sun in the recent past caused any significant perturbation of the new Oort Cloud comets. To investigate the origin of this apparent conflict, we have to see whether the responsible stars behind the currently observed comets may be hiding from discovery along with most other stars that recently encountered the Sun.

\subsection{Observational data}

The identification of previous close encounters between other stars and the Sun has been discussed by, for example García-Sánchez et al. (2001); Dybczyński \& Kwiatkowski (2003); Dybczyński (2006). The following discussion repeats some points made in these papers.

As a simplified approach, we judge the observability of stars and the access to relevant data on their positions and velocities by considering two space missions - one past and one future. The ESA HiPPARcos mission (1989-1993) represented a milestone in the mapping of the solar Galactic neighbourhood. It led to much improved parallaxes and proper motions of good quality for about 120000 stars (Perryman et al. 1997) and revolutionized searches for recent close encounters of stars with the Solar System (García-Sánchez et al. 2001). However, its limited magnitude coverage makes it reasonably complete only within a minute volume around the Sun relative to the extent of the Galaxy. A new revolution is expected from the ESA Gaia mission, to be launched in 2012. This will survey significant volumes with respect to all stellar types considered as Oort Cloud perturbers in this paper, including both accurate proper motion measurements and - with the aid of ground-based follow-up programmes - good-quality radial velocities.
Hipparcos observed all the stars with visual magnitude $V<$ 7.5-9 (depending on direction), and had a limiting magnitude $V=13$ (García-Sánchez et al. 2001). In our experiments, we assume that Hipparcos observed all stars with $V<8$ (independent of direction) and use a linearly decreasing detection probability between $V=8$ and $V=13$. We also consider that HiPPARcos was able to measure proper motions larger than the limit accuracy, i.e., $1 \mathrm{mas} / \mathrm{yr}$. Radial velocities were not observed by HIPPARcos but could often be found from the literature for the relatively bright stars in question (Dybczyński 2006).

For Gaia, the magnitude criterion is $V<20$, and we can assume that detection is practically complete everywhere on the sky for stars brighter than this limit. The parallax accuracies, as currently specified, are such that all stars within $500 \mathrm{pc}$ will have their parallaxes accurately measured, including almost all recent Oort Cloud perturbers. Moreover, although somewhat dependent on stellar spectral type, these accuracies can be used to deduce the limits of detectability for proper motions of $4 \mu$ as /yr for $V<$ $12,10 \mu \mathrm{as} / \mathrm{yr}$ for $12<V<17$, and $160 \mu \mathrm{as} / \mathrm{yr}$ for $17<V<20$.

The radial velocities measured by Gaia or later merit special attention. A relative error of $\sim 10 \%$ is a reasonable goal in order to identify whether a given star has passed near the Oort Cloud or not, and this translates into a few $\mathrm{km} / \mathrm{s}$ for most stars. Even though Gaia is expected to measure radial velocities, this goal will not be easy for early-type stars (B or A), whose spectra have only few and broad spectral lines. Hence, we may only be able to study stars with magnitudes as bright as $V<8$, and early-type stars more distant than 100 pc may not be able to have Gaiameasurable radial velocities. The later types on the other hand can be both relatively close and relatively faint at the same time. It appears that Gaia can then reach the required radial-velocity accuracy only down to $V \sim 14$, thus again excluding most of the detected stars.

We now consider the problem of identifying the possible Oort Cloud perturbers that have affected the currently observed new comets. We assume that all stars in our simulations are of this category $\left(d_{\min }<4 \times 10^{5} \mathrm{AU} \approx 2 \mathrm{pc}\right)$. In a forthcoming paper, we will examine in more detail the possibility of using more restrictive criteria. The stars of interest must have passed during the last revolution of the new comets with the longest periods. Since our quiescent Oort peak is mostly contained within $a<60000 \mathrm{AU}$, we use a time interval extending $15 \mathrm{Myr}$ into the past. On the basis of the estimated stellar encounter frequency, we expect there to be $\sim 600$ such stars. Assume that a star is found at distance $d$, which is measured with good accuracy. If $d<2$ pc, the star must obviously be identified independently of its motion, but this is a very unlikely outcome - in practically all cases the observed stars are much farther away. In these situations, the radial velocity $v_{\mathrm{r}}$ carries important information. If $v_{\mathrm{r}}<0$, the star is approaching and may thus be discarded. For $v_{\mathrm{r}}>0$, we may compute a good estimate of the time since closest approach $\left(t_{\min }\right)$ from

$t_{\min } \approx \frac{d}{v_{\mathrm{r}}}$

and if $t_{\min }>15 \mathrm{Myr}$, the star can be discarded. Otherwise the proper motion $\mu$ may be used to estimate $d_{\min }$ from

$d_{\min } \approx \frac{\mu d^{2}}{v_{\mathrm{r}}}$

using $v_{\mathrm{t}}=\mu d$ for the transverse velocity.

If both $v_{\mathrm{r}}$ and $\mu$ are known, it is thus a trivial decision about whether to earmark the star. However, since these stars are only 
A\&A 535, A86 (2011)

Table 1. Absolute visual magnitudes for the 13 different spectral types used in our simulations.

\begin{tabular}{lcccccccccccc}
\hline \hline B0 & A0 & A5 & F0 & F5 & G0 & G5 & K0 & K5 & M0 & M5 & gi & wd \\
\hline-2.95 & 0.55 & 1.85 & 3 & 3.8 & 4.35 & 5.1 & 5.95 & 7.25 & 9 & 13.95 & 1 & 12 \\
\hline
\end{tabular}

Table 2. Statistics on the stars perturbing injected comets during their last orbital period.

\begin{tabular}{lccccc}
\hline \hline Stars & All & 10 best & 5 best & 2 best & Best \\
\hline Gaia & $70 \% ; 96 \%$ & $85 \% ; 90 \%$ & $85 \% ; 88 \%$ & $86 \% ; 85 \%$ & $87 \% ; 84 \%$ \\
Hipp & $9 \% ; 74 \%$ & $22 \% ; 38 \%$ & $25 \% ; 33 \%$ & $26 \% ; 26 \%$ & $26 \% ; 20 \%$ \\
$\left\langle V_{\star}\right\rangle\left(\mathrm{km} \mathrm{s}^{-1}\right)$ & 52.8 & 44.2 & 43.3 & 42.3 & 42.0 \\
$\left\langle M_{\star}\right\rangle\left(M_{\odot}\right)$ & 0.46 & 0.90 & 1.00 & 1.07 & 1.09 \\
\hline
\end{tabular}

Notes. "All" refers to all the stars, "10 best" to the stars responsible for the ten largest negative perturbations of the perihelion distances, etc. Line "Gaia" gives the mean percent of stars detected by Gaia (first number) and, among the detected stars, the proportion for which the proper motion is above the corresponding limit accuracy of Gaia (second number). Line "Hipp" gives the same values for HipPARcos. The two last lines give the mean of the stellar encounter velocity and stellar mass.

a minority, we have to consider the case where either or both are unknown. If only $v_{\mathrm{r}}$ is unknown, one may use a likely upper limit such as, e.g., $100 \mathrm{~km} \mathrm{~s}^{-1}$ (see Dybczyński 2006) and thus obtain a minimum value of $d_{\mathrm{min}}$. Stars that are found to have certainly passed beyond 2 pc may then be discarded, while the remaining ones will be considered as suitable targets for radial velocity observations.

The procedure just described is the motivation behind using proper motion information in the identification process. The proper motion serves to discard stars for which no radial velocity observations have to be carried out. If on the other hand neither $v_{\mathrm{r}}$ nor $\mu$ is known, $\mu$ must be smaller than the above-mentioned limits and there is no way to exclude the possibility of a very close encounter.

To characterize this case, we estimate $\mu_{\max }$ by inserting $\left(v_{\mathrm{r}}\right)_{\max }=100 \mathrm{~km} \mathrm{~s}^{-1}$ into Eq. (4). For $d=100 \mathrm{pc}$, we then obtain $\mu_{\max } \approx 4$ mas/yr. Since the majority of HipPARcos-detected stars are closer than this, an undetected proper motion almost necessarily means that the star is a possible Oort Cloud perturber. For Gaia, the proper motion detection limits are smaller, and out to about $500 \mathrm{pc}$ an undetectable proper motion will always mean that the star is an interesting candidate for a radial velocity measurement, but we note that this will only yield a maximum value for the closest approach distance.

\subsection{Simulations}

For each injected comet in our simulations, we study all the stars that encountered the Oort Cloud during the last revolution of the comet, and for each star we compute its visual magnitude $V$, its proper motion, and its radial velocity at the time when the comet reaches its observable perihelion. In performing these calculations, we use the straight-line approximation rather than tracing the actual Galactic orbits. This is justified for time intervals of just a few Myr but may lead to significant errors in cases of >10 Myr. For the absolute magnitudes, we use the values given in Table 1 for the different spectral types. Every star considered here is regarded as an Oort Cloud perturber, although the actual perturbations affecting the injected comets are mostly very small.

From the calculated $V$ magnitude, we determine whether the star would have been detected by Hipparcos assuming that the comet is passing its perihelion at the present time, and whether it will be detected by Gaia. We also calculate the proper motion and determine whether this would have been measurable by
HipPARcos and will be measurable by Gaia. Finally, for the radial velocity we consider two scenarios for Gaia. According to the "optimistic" case, the radial velocities will be measurable for all stars that could possibly be Oort Cloud perturbers, while in the "pessimistic" scenario no velocities will be attainable from the ground, and the magnitude limits for Gaia-observed radial velocities will apply, as given above.

Table 2 presents some statistics on the stars perturbing the quiescent injected comets during their last orbital period. We list the fraction of stars detected by Gaia and Hipparcos and, for the detected stars, the fraction for which the proper motion is accessible. The mean velocity and mass of the perturbing stars is also given. Each quantity is listed first for all the perturbing stars, and then for only the stars with the ten, five, two, and one largest negative perturbations of the perihelion distance.

Looking at the entries for all the stars in Table 2, we note that HipParcos detects only $9 \%$ of the perturbing stars, whereas Gaia should detect $71 \%$ of these stars. Since the current stellar observations rely mainly on the HiPPARcos catalogue, most close-encounter stars are thus being missed. If we instead focus on the stars that provide the most important contributions to the decrease in the perihelion distance, these detection percentages increase for both Gaia and Hipparcos. For the latter, detection rises from $22 \%$ (for the ten most effective stars) to $26 \%$ (for the single most effective star), and Gaia detection similarly rises from $85 \%$ to $87 \%$. These increases in the detection probability are caused by the stars that have the greatest effect on the perihelion distances being those of both low velocity and high mass. They will have bright apparent magnitudes, because they are close by (owing to the small velocity) and luminous (owing to the large mass). We indeed note in Table 2 that the mean velocity decreases and the mean stellar mass increases, when going from all stars to the 10 best, 5 best, 2 best, and the very best among the stars (in terms of their contributions to the decrease in perihelion distance).

We also note that, because of its very high astrometric accuracy, Gaia will be able to measure the proper motions of the vast majority of Oort Cloud encountering stars, while the performance of HIPPARcos was not as good in spite of the detected stars being on the average closer ${ }^{3}$. Counting all the stars, the fraction with proper motion measurements is $96 \%$ for Gaia and $74 \%$ for Hipparcos. Focussing on the most effective perturbing

\footnotetext{
3 The most accurate proper motions currently available do not use only HiPPARCos data but also ground-based astrometry obtained far earlier.
} 


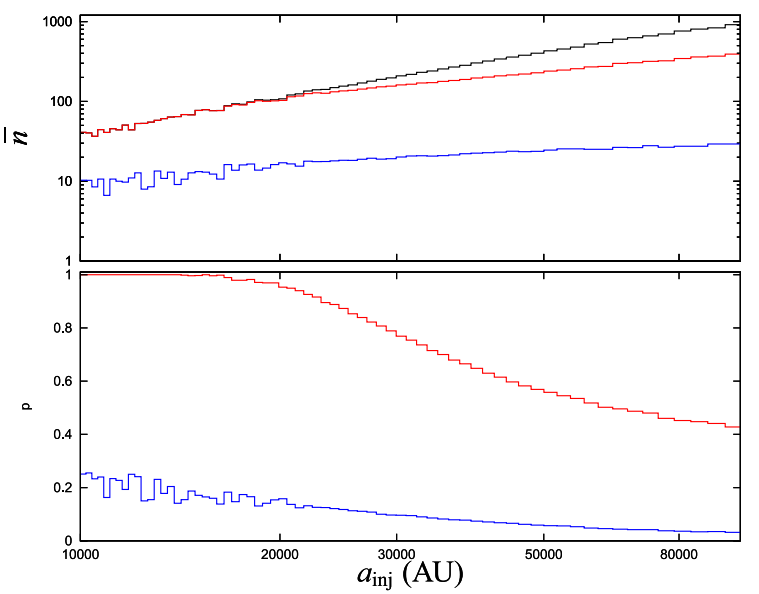

Fig. 8. Top: mean number of perturbing stars during the last orbital period of injected comets versus the semi-major axis at injection (black line) and number of perturbing stars detected by Gaia (red line) and Hipparcos (blue line). Only quiescent comets are considered. Bottom: fraction of perturbing stars observed by Gaia (red line) and HipParcos (blue line) versus the semi-major axis at injection.

stars, i.e., the closest and slowest encounters, the fraction decreases for both Gaia and Hipparcos, but while it falls all the way to $\sim 20 \%$ in the Hipparcos case when zooming in on the best star, for Gaia it remains above $80 \%$. Regarding radial velocities, these velocities were often available in the literature for Hipparcos stars, and for Gaia stars even in the pessimistic case, the fraction of detected stars for which the radial velocity is measured is $35 \%$ according to our simulations.

\subsection{Detection efficiencies}

The probability of the detection by each satellite strongly depends on the semi-major axis at injection $\left(a_{\text {inj }}\right)$. This is because with increasing orbital period, the time from stellar encounter to cometary perihelion statistically increases, so that the stars will tend to be more distant and thus fainter at the time of comet injection. Figure 8 shows, versus $a_{\text {inj }}$, the mean number of stars perturbing the quiescent injected comets during their last orbital period (this varies more or less as $a_{\mathrm{inj}}^{3 / 2}$ ), the mean numbers of these stars detected by Gaia and Hipparcos, and the corresponding fractions of detected stars in the Gaia and Hipparcos cases. We see how the probability of detection decreases with increasing semi-major axis. We note that Gaia detects all the perturbing stars for comets with $a<15000 \mathrm{AU}$ and $78 \%$ of the perturbing stars for $a \sim 30000 \mathrm{AU}$. The detections by HIPPARcos are never complete with only $20 \%$ of stars detected for $a \sim 15000 \mathrm{AU}$ and $10 \%$ for $a \sim 30000 \mathrm{AU}$.

Figure 9 is another clear indication of the improvements to be expected from Gaia. The diagrams show, for four different instants chosen at random during the last $3 \mathrm{Gyr}$ of our simulations, the stars that passed within $400000 \mathrm{AU}$ at times no later than $3 \mathrm{Myr}$ before that instant. This time interval corresponds to the orbital period of comets with $a \sim 20000$ AU. For larger semimajor axes, the relevant stars for injection could have passed further back in time. The four random instances are interpreted as the perihelion times of injected comets.

For each star, the closest encounter distance is plotted versus the time of closest encounter measured from the cometary perihelion time $(t=0)$. When we find the star to be detectable by Hipparcos, we indicate it with a sky-blue dot (left panels), and detectability by Gaia is marked by red dots (right panels). In each diagram, we have also plotted these data for the real stars found by Dybczyński (2006) as a representation of the best that could be done with the Hipparcos data. We have also indicated at which distance a comet on a parabolic or elliptic orbit would have been as a function of time, if it reaches perihelion at time zero. The "elliptic" curve corresponds to aphelion passage at the times in question.

The number of stars detected by Hipparcos is clearly very small, and we see that the work of Dybczyński (2006) corresponds well to the criteria used in our simulation. Recent encounters are strongly preferred, and thus the most effective stars are generally missed. For encounters further back in time, the stars tend to have moved to distances beyond the detectability limit of Hipparcos. On the other hand, the diagrams for Gaia illustrate the hope that one can place in this mission, when it comes to clarifying the origin of the observed, new Oort Cloud comets.

Finally, we performed the integration of the last orbital period of injected comets once more, taking into account only the stars detectable by either Hipparcos or Gaia. Figure 10 shows the number of quiescent comets that become injected versus the semi-major axis $a_{\text {inj }}$, taking into account all the stars, only the HipParcos stars, and only the Gaia stars. We have also plotted the fraction of injected comets using each sub-sample of stars with respect to the case where all the stars are taken into account.

Using only the stars detectable by HiPPARcos, we obtained a flux of injections that is less than half of the total flux, except near the maximum at $a_{\text {inj }} \sim 33000 \mathrm{AU}$, where the Hipparcos stars and the tides are able to inject a little more than $65 \%$ of the total. We note, however, that most of these injections are essentially tidal as in case $1 a$ of Fig. 3.

The calculations taking into account only the Gaiadetectable stars show that we have more than $80 \%$ of the total quiescent flux for $a_{\text {inj }}<40000$ AU. Then, however, the flux decreases to less than $10 \%$ of the total for $a_{\text {inj }} \sim 100000$ AU. Nevertheless, since the observed Oort spike is certainly situated mainly at $a<40000 \mathrm{AU}$, one can hope that, when the Gaia mission is over, it will be possible to simulate the last period of the observed comets far more reliably than one can do with the current data.

The fall-off in both Hipparcos and Gaia relative fluxes toward very large semi-major axes illustrates an important feature of comet injection dynamics. In Fig. 4, we saw that practically all the injected comets with $a_{\text {inj }}>50000$ AU belong to the $\mathbb{G}$ set, and this means that they might have been injected by the Galactic tides alone. However, as noted above, this result carries no information on the relative roles of stars and tides in the real injections. What we can now see in Fig. 10 is that the stars play a decisive role in comet injection at large semi-major axes, because even the removal of a small fraction of them mostly inhibits the injections that were found using the entire sample.

\section{Summary and conclusions}

We have performed three simulations of the evolution of the Oort Cloud, using one million test comets in each case. Perturbations due to the Galactic tides and passing stars have been incorporated. The three simulations differ in the selection of initial orbits and the sequence of stellar encounters. Each simulation extends over $5 \mathrm{Gyr}$, and during the last $3 \mathrm{Gyr}$ we register over 20000 comet injections, i.e., comets passing from perihelia $q>15$ AU to $q<5 \mathrm{AU}$ in one revolution. We have analysed in detail which events took place during these final revolutions 

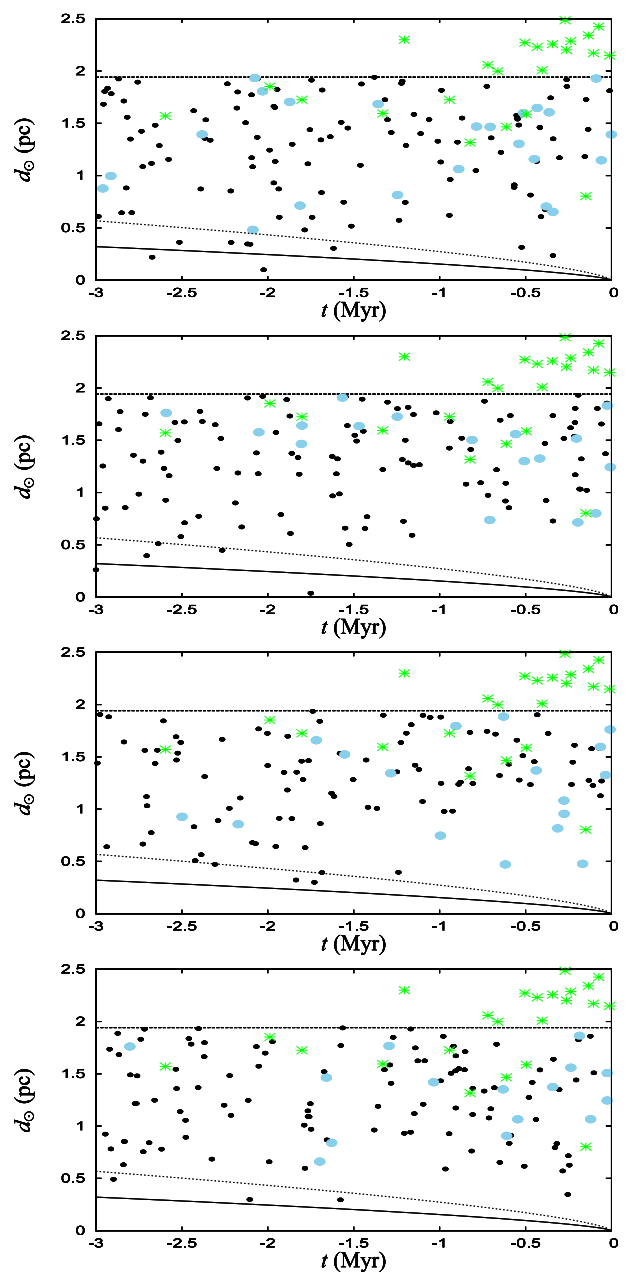
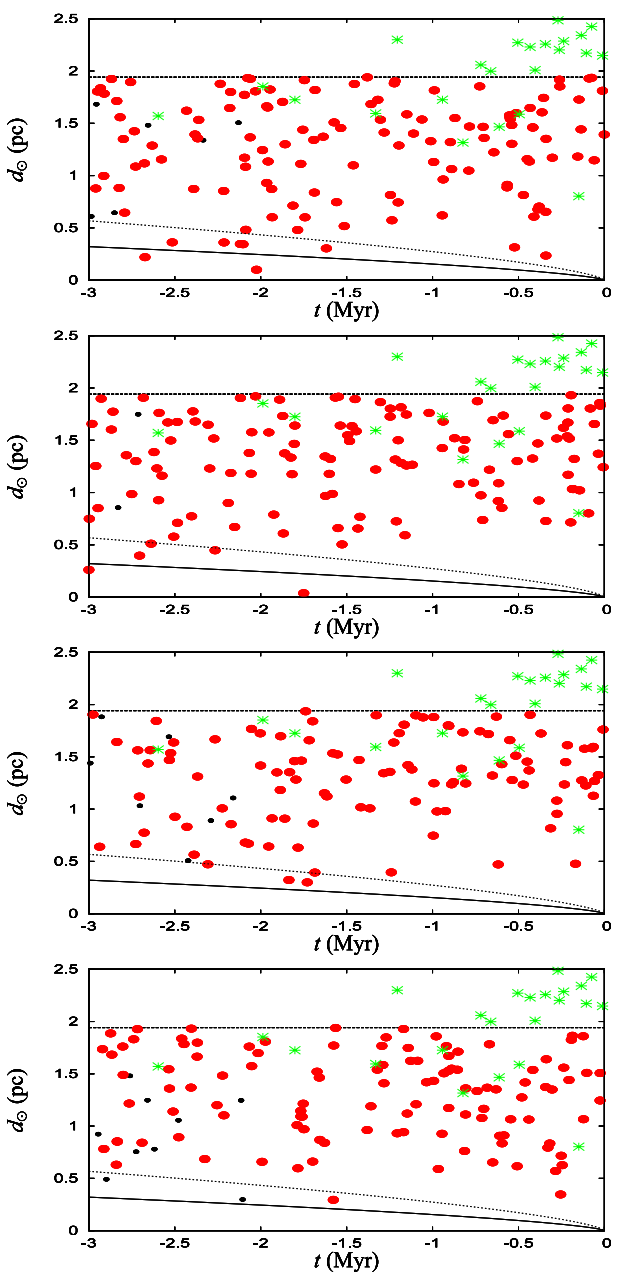

Fig. 9. For four different instants (one per line), we plot the encounter distance of stars versus the time of encounter counted from the perihelion passage of an injected comet $(t=0)$. The two curves indicate at which distance the comet would have been on a parabolic (dotted) or elliptic orbit (solid; the comet being at aphelion in this case). The black dots correspond to all the simulated stars passing during the $3 \mathrm{Myr}$ time span. In the left column, the sky-blue dots show the stars detected by Hipparcos. The same is done using red dots in the right column for the stars detected by Gaia. The green asterisks indicate the real stars identified by Dybczyński (2006). His sample extends to larger encounter distances than those of our simulation.

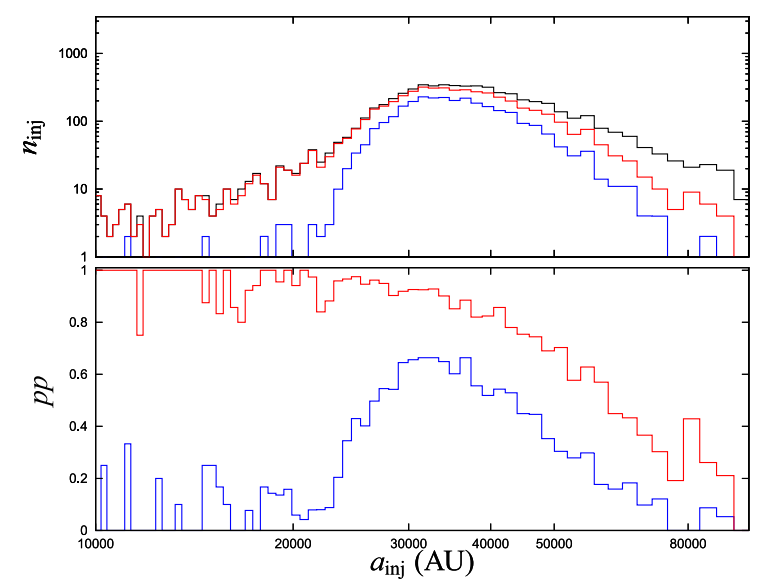

Fig. 10. Top: number of injected quiescent comets versus the semimajor axis at injection taking into account all the stars (black line), only the Hipparcos stars (blue line), and only the Gaia stars (red line). Bottom: fraction of injected comets using each sub-sample with respect to the all stars sample.

and assessed the roles played by the Galactic tides and by the passing stars.
One new feature of our analysis is the way in which we distinguish between cometary showers and the intervening, quiescent periods. We do not divide the time axis into shower and quiescent periods but instead classify each injected comet according to whether its last revolution could have been affected by an efficient, enhancement-producing stellar encounter or not. Our criterion has been chosen to be conservative in the sense that quiescent comets have to be injected only under the influence of stars with unremarkable injection efficiencies.

For quiescent comets, our simulated Oort peak agrees with the shapes found in other simulations with a maximum at a semimajor axis of about $33000 \mathrm{AU}$. We have found that the sharp decline in the injected flux with increasing semi-major axis beyond the maximum is consistent with the increasing difficulty in sending a comet to perihelion when the perihelion distance is shorter than the observable limit. The distribution for nonquiescent comets is quite flat, as expected for a situation where the loss cone is filled at practically any semi-major axis.

We have defined two sets of injected comets according to whether the post-injection orbit could have been reached by a purely tidal injection ( $\mathbb{G}$ set) or whether an actual jump from $q>15$ AU to $q<5$ AU was caused by a stellar encounter ( $\mathbb{S}$ set). The smallest semi-major axis found in the $\mathbb{G}$ set is 
about $23000 \mathrm{AU}$, which is the limit below which it is impossible for the Galactic tides to inject comets on their own. On the other hand, for semi-major axes larger than $50000 \mathrm{AU}$ almost all the injected comets belong to the $\mathbb{G}$ set, which is unsurprising, because for these semi-major axes the tides will always have time to remove the perihelion from the observable zone during one orbital revolution of the comet. We conclude that the tides, in principle, could have injected the comets, but that it is impossible to state whether they actually did so. We argue that owing to the short timescales and large perturbations involved, comet injection from the outer part of the Oort Cloud is quite a precarious operation, involving a fine tuning of stellar and tidal perturbations.

The $\mathbb{S}$ set comprises essentially all the injections at semimajor axes of about $10000 \mathrm{AU}$, which shows the predominance of stellar injections in this range, but drops to smaller fractions around $15000 \mathrm{AU}$. We have identified a range of semi-major axis, extending from $\sim 15000 \mathrm{AU}$ to $\sim 45000 \mathrm{AU}$, where the union of the $\mathbb{S}$ and $\mathbb{G}$ sets fails to cover the ensemble of all injected comets - indicating that a synergy is at work between the two perturbers. This synergy is found to reach its maximum close to the critical limit of tidal injections, where as much as $70 \%$ of the actual injections can be ascribed to the synergy, and extends across most of the Oort peak.

We also investigated whether the inclusion of stellar perturbations during the last revolution has either a positive or negative effect on the number of injected comets. The result is that the overall effect is positive because of an increase in the injection efficiency inside and at the inner part of the Oort peak, while at the outer part the differences are very small.

After demonstrating that passing stars should have had a large influence on most of the injections responsible for the observed new Oort Cloud comets, we checked the observability of those stars using our simulations by computing the magnitude, proper motion, and radial velocity of each perturbing star at the moment the injected comet reaches perihelion. We then compared these results with the detectability limits of both HIPPARcos and Gaia. One result that we found is that Hipparcos is mostly unable to detect the perturbing stars, even when we concentrated on the stars causing the largest decrease in perihelion distance, while Gaia is likely to detect nearly all the most efficient perturbers and most of the perturbers at large. We have thus shown why Dybczyński (2006) was unable to identify the stellar perturbations that affected the new comets, while confirming that his search for stars using Hipparcos data was about as good as one can reasonably expect. We also note that using only the stars that Hipparcos could have detected and the tides, our simulated flux of quiescent injected comets is much reduced.

The proper motions of the perturbing stars have been generally difficult to obtain using HipParcos data but will become accessible in the vast majority of cases using Gaia. Regarding the radial velocities, Gaia will provide them in many cases, and we argue that in the remaining cases, these velocities will likely be attainable using ground-based follow-up observations. Therefore, the prospects for clarifying the origin of the new comets after Gaia look rather good.

Finally, we comment on what is lacking in our model. We note that we have only studied the present situation, using the Galactic tides and stellar perturbations typical of the Sun's present position in its Galactic orbit, while owing to radial and vertical orbital oscillations of the Sun, large changes may be expected with time (Gardner et al. 2011). Since our study has not been concerned with comet injection at other times in the history of the Solar System, this may not be a large problem, but obviously the past motion of the Sun - including possible encounters with GMCs as well - may have left its imprints on the structure of the Oort Cloud in ways that our model does not account for.

A potentially more serious problem is that our model of comet injection does not incorporate a realistic treatment of planetary perturbations. For instance, it may not be in reality impossible for comets to enter into orbits with perihelion distances shorter than $5 \mathrm{AU}$ via a preceding passage with $q \sim 10 \mathrm{AU}$. Kaib \& Quinn (2009) started to investigate this problem, but their model did not include the stellar perturbations during the analysed time interval except as a check, and we propose that a careful study of the complete dynamics of comet injection will be needed to reach safe conclusions. We intend to perform such a study in the near future, and the present one will then serve as a useful comparison case.

Acknowledgements. We gratefully acknowledge the remarks made by Ramon Brasser and an anonymous referee, which greatly helped to improve the quality of the paper. This work was possible thanks to research grants from the Polish Government (NN 203392 734) and the Swedish National Space Board $(119 / 07)$. The work of G.B.V. has also been supported by the Italian Space Agency, under contract ASI/INAF I/015/07/0.

\section{References}

Breiter, S., Fouchard, M., Ratajczak, R., \& Borczyk, W. 2007, MNRAS, 377, 1151

Delsemme, A. H. 1987, A\&A, 187, 913

Dones, L., Weissman, P. R., Levison, H. F., \& Duncan, M. J. 2004, Comets II, 153

Duncan, M., Quinn, T., \& Tremaine, S. 1987, AJ, 94, 1330

Dybczyński, P. A. 1994, Celest. Mec. Dyn. Astron., 58, 139

Dybczyński, P. A. 2001, A\&A, 375, 643

Dybczyński, P. A. 2002, A\&A, 396, 283

Dybczyński, P. A. 2006, A\&A, 449, 1233

Dybczyński, P. A., \& Kwiatkowski, T. 2003 [arXiv: astro-ph/0307019v3]

Dybczyński, P., \& Królikowska, M. 2011, MNRAS, 416, 51

Emel'yanenko, V. V., Asher, D. J., \& Bailey, M. E. 2007, MNRAS, 381, 779

Fouchard, M., Froeschlé, Ch., Breiter, S., et al. 2007, Lect. Not. Phys., 729, 271

Fouchard, M., Froeschlé, Ch., Rickman, H., \& Valsecchi, G. B. 2010, Lect. Not. Phys., 790, 401

Fouchard, M., Froeschlé, Ch., Rickman, H., \& Valsecchi, G. B. 2011, Icarus, 214,334

Francis, P. J. 2005, ApJ, 635, 1348

García-Sánchez, J., Preston, R. A., Jones, D. L., et al. 1999, AJ, 117, 1042

García-Sánchez, J., Weissman, P. R., Preston, R. A., et al. 2001, A\&A, 379, 634

Gardner, E., Nurmi, P., Flynn, C., \& Mikkola, S. 2011, MNRAS, 411, 947

Heisler, J., \& Tremaine, S. 1986, Icarus, 65, 13

Hills, J. G. 1981, AJ, 86, 1730

Holmberg, J., \& Flynn, C. 2000, MNRAS, 313, 209

Kaib, N. A., \& Quinn, T. 2009, Science, 325, 1234

Królikowska, M. 2006, Acta Astron., 56, 385

Królikowska, M., \& Dybczyński, P. 2010, MNRAS, 404, 1886

Oort, J. H. 1950, Bull. Astron. Inst. Neth., 11, 91

Öpik, E. 1932, Proc. American Academy of Arts and Science, 67, 139

Perryman, M. A. C., Lindegren, L., Kovalevsky, J., et al. 1997, A\&A, 323, L49

Rickman, H., Froeschlé, Ch., Froeschlé, Cl., \& Valsecchi, G. B. 2004, A\&A, 428,673

Rickman, H., Fouchard, M., Valsecchi, G. B., \& Froeschlé, Ch. 2005, Earth Moon and Planets, 97, 411

Rickman, H., Fouchard, M., Froeschlé, Ch., \& Valsecchi, G. B. 2008, Cel. Mech. Dyn. Astron., 102, 111

Wiegert, P., \& Tremaine, S. 1999, Icarus, 137, 84 